\title{
Diagonal stripes in the spin glass phase of cuprates
}

\author{
G. Seibold ${ }^{\mathrm{a}}$, J. Lorenzana ${ }^{\mathrm{b}}$ \\ ${ }^{a}$ Institut für Physik, BTU Cottbus, PBox 101344, 03013 Cottbus, Germany \\ ${ }^{b}$ SMC-INFM-CNR and Dipartimento di Fisica, Università di Roma "La Sapienza”, P.le Aldo Moro 5, I-00185 Roma, Italy
}

\begin{abstract}
Based on the unrestricted Gutzwiller approximation we study the possibility that the diagonal incommensurate spin scattering in the spin glass phase of lanthanum cuprates originates from stripe formation. Similar to the metallic phase two types of diagonal stripe structures appear to be stable: (a) site-centered textures which have one hole per site along the stripe and (b) ferromagnetic stair-case structures which are the diagonal equivalent to bond-centered stripes in the metallic phase and which on average have a filling of 3/4 holes per stripe site. We give a detailed analysis of the stability of both diagonal textures with regard to the vertical ones.
\end{abstract}

Key words: Cuprate superconductors, Stripes, Gutzwiller approximation

In lanthanum cuprates (LSCO) the low energy incommensurate magnetic scattering shows an interesting behavior upon entering the insulating phase, i.e. below doping $x_{c} \approx 0.055$. According to the experimental findings [1, 2, 3, 4] the incommensurability $\delta$ (defined as the deviation of the magnetic peak from $Q_{A F}$ ) rotates from vertical above $x_{c}$ to diagonal below $x_{c}$ where it is even detected in the elastic channel. Moreover, the orthorhombic lattice distortion allows one to conclude that the diagonal magnetic scattering is one-dimensional with the associated modulation along the orthorombic aaxis. Contrary, in the vertical phase the twinned LSCO samples show a two-dimensional scattering geometry and the one-dimensionality of the stripe modulations is only suggested by additional arguments. The magnitude of the incommensurability is linear in doping $\delta=x$ also across the rotation but approaches $\delta=x / 2$ upon approaching the border of the AF phase at $x=0.02$. Here we investigate the static properties of diagonal stripe textures based on the Gutzwiller approximation (GA) of the one-band Hubbard model. We use the following parameter set for the Hubbard model which fit the magnon dispersion of undoped LCO and several other properties [5]: On-site repulsion $U=7.5 \mathrm{eV}$, Nearestneighbor hopping $t=340 \mathrm{meV}$, Next-nearest neighbor hopping $t^{\prime}=-0.2 t$.

Fig. 1a,b shows the two diagonal stripe textures which we found to be stable saddle-points of our GA calculations. First we have the diagonal site-centered (SC) texture (Fig. 19) where the AF order-parameter Preprint submitted to Elsevier changes sign on the stripe sites similar to the corresponding vertical structure in the metallic phase. Second we find a stair-case structure (Fig. 13) with the sign change occuring on the bonds. Contrary to vertical bond-centered textures (BC) the diagonal topology implies a net ferromagnetic moment of the stripes. Note that diagonal BC (DBC) stripes which are separated by an even number of unit cells along $x$ (or $y$ ) have opposite magnetization whereas for distances with an odd number of unit cells all stripes have the same moment so that the layer becomes macroscopically ferromagnetic. Fig. 1t reports the energy per added hole, $e_{h}=\left[E\left(N+N_{h}\right)-E(N)\right] / N_{h}$ vs. the stripe filling $v=N_{h} / N_{s}$. Here $N_{h}$ denotes the number of doped holes, $N$ is the number of sites and $N_{s}$ the number of domain walls. For comparison we also show the corresponding curve for a vertical SC texture (the vertical BC is identical). Note that the curves in Fig. 16 correspond to low doping results in the sense that interaction effects between stripes are negligible. From Fig. 1t it turns out that the preferred filling of vertical stripes $\left(v_{o p t} \approx 0.55\right)$ increases to $\left(v_{o p t} \approx 0.75\right)$ for DBC and $\left(v_{o p t} \approx 1\right)$ for DSC stripes. In Ref. [6] we have shown that this can account for the doping dependent incommensurability as observed experimentally [1, 2, 3, 4].

In order to analyze the differences between the different diagonal configurations and between vertical and diagonal stripes we follow the procedure detailed in Ref. [7] and expand $e_{h}$ in a power series in $v$ :

$$
e_{h}=A / v+B+C v \text {. }
$$



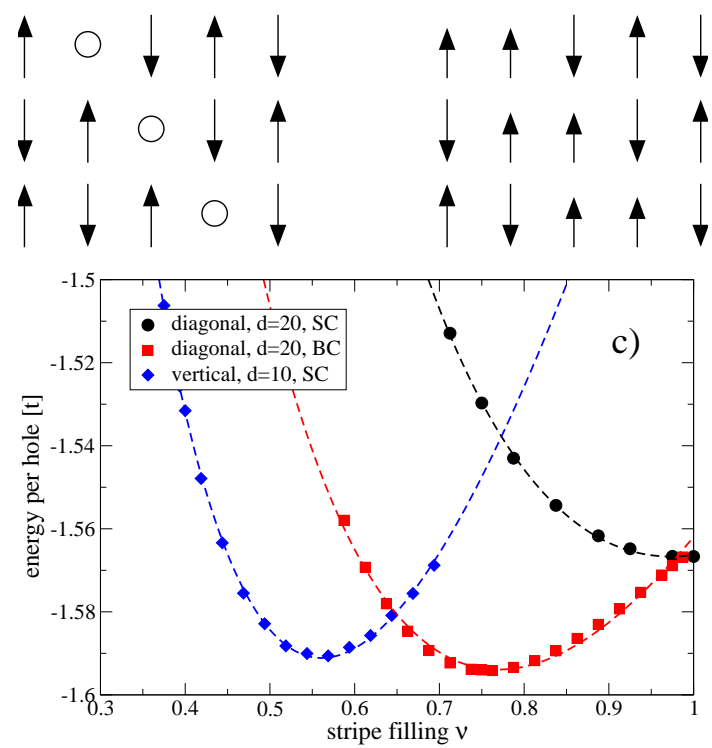

Figure 1: Diagonal stripe textures investigated in this paper: (a) sitecentered stripe where non-magnetic sites are indicated by a circle and (b) stair-case (bond-centered) stripes. (c) Binding energy per hole as a function of stripe filling for diagonal SC (circles), diagonal BC (squares) and vertical SC (diamonds) stripes. The distance $d$ between stripes corresponds to the separation of the domain walls along the $x-$ or $y$-direction. The dashed lines are fits as explained in the text.

As can be seen from the dashed lines in Fig. 1t this relation provides an excellent fit to the data points for both vertical and diagonal stripes. The coefficients in the expansion can be interpreted as follows [7]: $A$ is the energy cost per stripe unit to create an antiphase domain wall in the undoped (i.e. AF) system. The parameter $B$ corresponds to the chemical potential to add one hole into the empty stripe, and $\mathrm{C}$ is related to the inverse local compressibility incorporating kinetic and interaction energy contributions but fixing the stripe separation.

For undoped SC stripes the chemical potential is at the center of an active band (between lower and upper Hubbard band) which width is $\sim z^{2} W^{0}\left(t^{\prime}\right)\left(\sim z^{2} W^{0}(t)\right)$ for diagonal (vertical) SC stripes. Here $W^{0}$ denotes the width of an uncorrelated quasi one-dimensional band and $z^{2}$ is the Gutzwiller hopping renormalization factor which for fixed $U$ behaves as $z^{2}=1-\left(\alpha / W^{0}\right)^{2}$. Instead, for undoped BC diagonal stripes the associated ferromagnetic stripe magnetization cancels Gutzwiller band narrowing for the active band. In this case the chemical potential is on the top of the band with width $W^{0}\left(t^{\prime}\right)$. From these considerations we can understand the difference in the fitting parameters for the various textures which are reported in the table.

\begin{tabular}{|c|c|c|c|}
\hline & Vertical SC & Diagonal SC & Diagonal BC \\
\hline \hline A [t] & 0.29 & 0.53 & 0.36 \\
\hline B [t] & -2.62 & -2.66 & -2.52 \\
\hline C [t] & 0.92 & 0.56 & 0.60 \\
\hline
\end{tabular}

Table 1: Parameters (in units of $t$ ) obtained by fitting the $e_{h}(v)$ curves of $d=20 \mathrm{SC}$ and $\mathrm{BC}$ diagonal and $d=10$ vertical SC stripes to the expression Eq. 11. The resulting fits are shown in Fig. 1.

Creation of a vertical (diagonal) BC stripe in an ordered AF requires the destruction of one (two) AF bonds and the formation of one (two) ferromagnetic bonds per stripe unit (diagonal exchange couplings contribute only a fraction $\left(t^{\prime} / t\right)^{2}$ to the energy estimate and are neglected.) In the Néel limit the associated energy is $A_{\text {vert }}=J / 2$ and $A_{\text {diag }}=J$, respectively, which is further reduced due to quantum fluctuations. In case of SC stripes the argument becomes more subtle as explained in Ref.[7]. The essential point here is that the core behaves as a spin liquid which again leads to $A \sim J$.

The parameter $C$ corresponds to the relaxation energy upon adding holes to the undoped system and is decomposed into a kinetic and interaction contribution $C=C_{K}+C_{I} . C_{K}$ can be determined by optimizing the variational parameters for small $v$ and then computing $e_{h} v s . v$ without further changing the variational parameters, i.e. by solely filling the frozen bands. The resulting linear contribution in $v$ to $e_{h}$ gives the $C_{K}$ parameter, and since $C$ is known we also obtain $C_{I}$. From this analysis we find that the kinetic contribution to $C$ for SC diagonal stripes is practically zero due to the almost flat active band. For vertical SC and diagonal BC stripes the kinetic contribution in both cases is $C_{K} \approx 0.2 t$.

The almost vanishing $C_{K}$ parameter makes the diagonal textures more susceptible towards disorder, especially due to pinning by impurity dopants which tend to localize the holes. In fact, replacing $C u$ by nonmagnetic $\mathrm{Zn}$ ions [8] shifts the incommensurability close to the value predicted for SC diagonal stripes.

The cooperation of J.L. and G.S. is supported by the Vigoni foundation.

\section{References}

[1] S. Wakimoto et al., Phys. Rev. B 60, R769 (1999).

[2] S. Wakimoto et al., Phys. Rev. B 61, 3699 (2000).

[3] M. Matsuda et al., Phys. Rev. B 62, 9148 (2000).

[4] M. Fujita et al., Phys. Rev. B 65, 064505 (2002).

[5] G. Seibold and J. Lorenzana, Phys. Rev. B73, 144515 (2006).

[6] G. Seibold and J. Lorenzana, Phys. Rev B (2009).

[7] G. Seibold and J. Lorenzana, Phys. Rev. B 69, 134513 (2004).

[8] M. Matsuda, M. Fujita, and K. Yamada, Phys. Rev. B 73, R140503 (2006). 